American Journal of Immunology 1 (2): 94-95, 2005

ISSN 1553-619X

(C) 2005 Science Publications

\title{
Botulinum Toxin as a Novel Addition to Anti-Arthritis Armamentarium
}

\author{
Hamid Namazi and Zahra Majd \\ Department of Orthopedic Surgery, Shiraz University of Medical Sciences, Iran
}

\begin{abstract}
Osteoarthritis is the most common joint disease and is among the most frequent health problems for middle aged and older people. There is strong evidence that proinflammatory cytokines contribute to cartilage degradation in osteoarthritis. Interleukin-1 is the prototypical proinflammatory cytokine implicated in the pathogenesis of cartilage matrix degeneration. Results from studies in animal models provide stronger evidence implicating a role for interleukin-1 in the pathogenesis of matrix loss in osteoarthritis. These include the induction of proteoglycan loss by intraarticular injection of interleukin-1 and the capacity of the inhibitor of interleukin-1, interleukin-1 receptor antagonist (IL-1 ra), to slow the progression of cartilage loss in animal models of osteoarthritis. The botulinum toxin has been used in many clinical situations such as: cerebral palsy, headache, cosmesis and etc. Moreover, there is evidence that botulinum toxin specifically inhibits Rho GTPase by ADP-ribosylation of aminoacid ASn-41. Rho GTPase is necessary for activation of interleukin-1 inflammation pathway.

Based on previously mentioned evidence we suggest, intraarticular injection of the botulinum toxin may be a useful therapy in osteoarthritis.
\end{abstract}

Key words: Botulinum toxin, arthritis, cytokine, GTPase

\section{INTRODUCTION}

A major challenge of contemporary medicine is to break the traditional compartmentalization that frequently separates various fields. Unexpected linkages between various areas of medicine are in deed of particular interest.

In this paper, the aim is to enlighten the orthopaedic surgeon colleagues about the recently discovered Immuno modulatory effects of botulinum toxin in order to encourage research on the use of this agent in the treatment of osteoarthritis.

Osteoarthritis (OA) is the most common joint disease and is among the most frequent and symptomatic health problems for middle aged and older people ${ }^{[1,2]}$. It is characterized by joint pain and dysfunction; and in its advanced stages, joint contractures, muscle atrophy and limb deformity ${ }^{[3]}$.

Decreasing the burden of osteoarthritis will require finding methods of preventing and slowing the joint degeneration that causes osteoarthritis and decreasing the joint pain and dysfunction.

The role of cytokines in cartilage degeneration in osteoarthritis: Interleukin-1 is the prototypical proinflammatory cytokine implicated in the pathogenesis of cartilage matrix degradation in osteoarthritis. The first description of a biologic activity that is attributable to IL-1 as a regulator of chondrocyte function comes from the studies of Fell et al. ${ }^{[4]}$. They used an in vitro model in which they cocultured healthy non inflamed procine synovial tissue with cartilage fragments. They noted the progressive break down of the cartilage matrix by the resident chondrocytes and based on these findings speculated that the synovial tissue was producing a soluble factor they termed catabolin that stimulated chondrocytes to break down their surrounding cartilage matrix. In parallel studies conducted in other laboratories, investigators identified similar activities in culture supernatants from mononuclear cells and synovium that could stimulate the production of proteolytic enzymes from cultured chondrocyte $^{[5-7]}$.

Subsequently, the purification and cloning of IL-1 permitted the definitive identification of this cytokine as one of the soluble factors with the capacity to induce chondrocyte mediated cartilage degradation ${ }^{[8,9]}$. Table 1 summarizes the findings supporting a role for IL-1 in the pathogenesis of cartilage matrix break down in OA.

These include the induction of proteoglycan loss by intra-articular injection of IL-1 and the capacity of the inhibitor of IL-1, interleukin-1 receptor antagonist (IL-1ra), to slow the progression of cartilage loss in animal models of osteoarthritis ${ }^{[10-12]}$. There is strong evidence that additional proinflammatory cytokines contribute to cartilage degradation in osteoarthritis. But interleukin-1 is 100 fold to 1000 -fold more potent on a molar basis than other cytokines ${ }^{[13,14]}$.

Botulinum toxin: Bacteria produce many enzymes that show extra ordinary specificity for mammalian intracellular proteins. The specificity of these bacterial enzymes has not only made them a valuable tool for elucidating the cellular functions of their targets but has

Corresponding Author: Hamid Namazi, M.D., Assistant Professor, Shiraz University of Medical Sciences, Department of Orthopaedic Surgery, Fax: 0711-6231410, Tel: 0711-6246093 
also increased our understanding of protein interactions ${ }^{[15]}$.

Clostridium botulinum is no exception, producing two classes of enzymes that have very specific protein targets, the neurotoixn A-G and the ADPribosyltransferases $\mathrm{C} 2, \mathrm{C} 3$ bot 1 and $\mathrm{C} 3$ bot $2^{[15]}$.

Table 1: Evidence that IL-1 plays a role in osteoarthritis (OA) pathogenesis

\begin{tabular}{ll}
\hline Patients with OA & Studies \\
\hline Detected in & Wood et al, \\
synovial fluid & Westacott et al. \\
Detected in synovium & myers et al, smith et al. \\
$\begin{array}{l}\text { Detected in cartilage } \\
\text { Animal models: Intra articular }\end{array}$ & Middleton et al, Towle et al. \\
$\begin{array}{l}\text { injection Stimulates cartilage loss } \\
\text { and intra articular IL-1ra }\end{array}$ & Cettipher et al. O'Byrne et al. et al, Pelletier et al. \\
reduces progression of early OA & \\
\hline
\end{tabular}

$\mathrm{C} 2$ and $\mathrm{C} 3$ bot are part of a larger family of ADPribosylating toxins, including diphtheria toxin and cholera toxin, which cleave NAD and transfer ADPribose to target proteins. Although the members of this family have homologous enzymatic domains and similar active sites, these toxins ADP ribosylate and therefore, disable a range of cellular targets ${ }^{[15]}$. Rho family GTPases control the assembly of both cellmatrix and cell-cell adhesion complexes. IL-1 receptor signaling complex contained these $\mathrm{G}$ proteins and Rho GTPase is an essential unit for activation of IL-1 inflammatory pathway. C3 transferase exoenzyme (Botulinum toxin) specifically inhibits Rho GTPase by ADP-ribosylation of amino acid ASN-41 ${ }^{[16,17]}$. Based on previously mentioned evidence we suggest, intraarti cular injection of the botulinum toxin may be a useful therapy in osteoarthritis.

\section{REFERENCES}

1. Brooks, P.M., 2002. Impact of osteoarthritis on individuals and society: How much disability? Social consequences and health economic implications. Curr. Opin. Rheumatol., 4: 573-577.

2. Leigh, J.P., W. Seavey and W. Leistiko, 2001. Estimating the costs of job related arthritis. J. Rheumatol., 28: 1647-1654.

3. Buckwalter, J.A. and H.J. Mankin, 1997. Articular Cartilage II. Degeneration and osteoarthrosis, repair, regeneration and transplantation. J. Bone Joint Surg., 79: 612-632.

4. Fell, H.B. and R.W. Jubb. The effect of synovial tissue on the break down of articular cartilage in organ culture. Arthritis. Rheum., 20: 1359-1371.

5. Dayes, J.M., R.G. Russell and S.M. Krone, 1977. Collagenase production by rheumatoid synovial cells: Stimulation by a human lymphocyte factors. Science, 195: 181-183.
6. McGurie-Goldring, M.B., J.E. Meats, D.D. Wood et al., 1984. In vitro activation of human chondrocytes and synoviocytes by a human interleukin-1 like factor. Arthritis. Rheum., 27: 654-662.

7. Meats, J.E., M.B. McGuire and R.G. Russel, 1980. Human synovium releases a factor which stimulates chondrocytes production of PGE and plasminogen activators. Nature, 286: 891-892.

8. Wood, D.D., E.J. Ihrie and C.A. Dinarello et al., 1983. Isolation of an Interleukin- 1 like factor from human joint effusions. Arthritis. Rheum., 26:975983.

9. Wood, D.D., E.K. Bayne and M.D. Goldring et al., 1985. The four biochemically distinct species of human interleukin-1 all exhibit similar biologic activities. J. Immunol., 134: 895-903.

10. Caron, I.P., J.C. Fernandes and J. Martel-pelletier et al., 1996. Chondroprotective effect of intraarticular injection of interleukin-1 receptor antagonist in experimental osteoarthritis. Suppression of collagenase-1 expression. Arthritis. Rheum., 39: 1535-1544.

11. Fernandes, J.C., J. Martel-Pelletier and J.P. Pelletier, 2002. The role of cytokines in osteoarthritis pathophysiology. Biorheology, 39: 237-246.

12. Pelletier, J.P., J.P. Caron and S.C. Evans et al., 1997. In vivo suppression of early experimental osteoarthritis by interleukin-1 receptors antagonist using gene therapy. Arthritis. Rheum., 40: 10121019.

13. Van den Berg, W.B., 2001. Anti-cytokine therapy in chronic destructive arthritis. Arthritis Res., 3: 1826.

14. Van den Berg, W.B., 2001. Uncoupling of inflammatory and destructive mechanisms in arthritis. Arthritis. Rheum., 30: 7-16.

15. Holbourn, K.P., J.M. Sutton and C. Shore et al., 2005. Molecular recognition of an ADP - ribosyl transferase; clostridium botulinum $\mathrm{C} 3$ exoenzyme. Proc. Natl. Acad. Sci., 102: 535-5364.

16. Harmey, D., G. Stenbeck and C.D. Nobes et al., 2004. Regulation of osteoblast differentiation by Pasteurella multocida toxin (PMT): a role for Rho GTP ase in bone formation. J. Bone Miner. Res., 19: 661-67.

17. Singh, R., B. Wang and A. Shirraikar et al., 1999. The IL-1 receptor and Rho directly associate to drive cell activation in inflammation. J. Clin. Invest., 103: 1561-1570. 